\title{
Clinical features, severity and outcome of adult inpatients with single and dual infections with influenza A (H1N1) and SARS-CoV-2: a retrospective study in China
}

\author{
Jiazhen Zheng ${ }^{1}$, Shan Huang ${ }^{1}$, Fengjuan Chen ${ }^{2}$, Keyi $\mathrm{Wu}^{1}$, Furong $\mathrm{Li}^{1}$, Jianyun $\mathrm{Lu}^{3}$, \\ Huamin Liu ${ }^{1}$, Rui Zhou ${ }^{1}$, Zhiwei Huang ${ }^{1}$, Bingyao Meng ${ }^{1}$, and Xianbo $\mathrm{Wu}^{1}$ \\ ${ }^{1}$ Southern Medical University \\ ${ }^{2}$ Guangzhou Eighth People's Hospital \\ ${ }^{3}$ Guangzhou Center for Disease Control and Prevention
}

June 26, 2020

\begin{abstract}
Background: The Northern Hemisphere faces the prospect of the coronavirus disease 2019 (COVID-19) pandemic and a simultaneous epidemic of seasonal influenza. However, the clinical impacts of single and dual infections with influenza A (H1N1) and SARS-CoV-2 haven't been well described. Objectives: We aim to compare the clinical features, severity and outcome among patients with single and dual infections with influenza A (H1N1) and SARS-CoV-2. Methods: Characteristics and outcomes in adults ([?]18 years) hospitalized for H1N1 infection $(\mathrm{n}=220)$ were retrospectively compared with cases hospitalized for SARS-CoV-2 infection $(\mathrm{n}=249)$ and co-infection with both viruses $(\mathrm{n}=36)$ in a single-center study in Guangzhou, China. Results: Compared with COVID-19 infection, patients with H1N1 were more likely to have clinical symptoms $(\mathrm{P}<$ $0.05)$, higher admission body temperature $(37.8$ vs $37.2, \mathrm{P}<0.05)$, but shorter duration of hospitalization $(7.0$ vs 17.5 days, $\mathrm{P}<0.05)$ and lower incidence of pneumonia during hospitalization $(46.8 \%$ vs $73.1 \%, \mathrm{P}<0.05)$. Co-infection tended to be more common among older patients (median age 56.0 years old) and was associated with increased odds of prolonged duration of hospitalization ( $>20 \mathrm{~d}$ ), pneumonia, secondary bacterial infections and admittance to ICU, compared with H1N1 patients $(\mathrm{P}<0.05)$. Conclusion: COVID-19 patients have a higher incidence of pneumonia during hospitalization whilst fewer onset symptoms than H1N1 patients. Co-infection was more found in older age and was associated with poorer clinical outcomes.
\end{abstract}

\section{Introduction}

Influenza is a contagious respiratory disease that is widespread across the globe. Despite advances in medical technology, influenza causes considerable hospitalizations and mortality ${ }^{1,2}$. H1N1 is a subtype of influenza A virus that leads to respiratory infections and has caused two pandemics over the past 102 years $^{3,4}$. The most recent pandemic of H1N1 influenza occurred in 2009 and affected 60.8 million people, resulting in 284,000 deaths worldwide ${ }^{5,6}$.

Severe acute respiratory syndrome coronavirus 2 (SARS-CoV-2) has now spread to most countries, with WHO declaring a COVID-19 pandemic on March 1, 20207. As of June 24, 2020, more than nine million confirmed cases and 473,061 deaths had been reported globally ${ }^{8}$. The outbreak of COVID-19 began in December 2019, which also corresponded with the flu season in China and the United States ${ }^{9,10}$, the management of the disease was often complicated by the diversity in "influenza-like" clinical manifestations. Hence, differentiating the clinical impact of SARS-CoV-2 and H1N1 virus-infection will facilitate rational arrangements for implementation of COVID-19 prevention and treatment strategies. Previous studies have only compared the general epidemiological and clinical presentation of patients infected SARS-CoV-2 and H1N1 virus ${ }^{11-16}$. 
However, few studies have been performed to compare the severity and clinical outcome of these two types of infection.

Recently, influenza A(H1N1)pdm09 was reported as a common co-pathogen in COVID-19 patients ${ }^{17}$, and the viral co-infection is proved to be associated with more adverse clinical outcomes ${ }^{18-20}$. The data on the clinical characteristics and outcomes of patients mix-infected with SARS-CoV-2 and H1N1 are scarce, but are of paramount importance to the development of efficacious interventions and protective measures. Therefore, to facilitate efforts, both in China and globally, we report the clinical features, severity and outcome of patients with laboratory-confirmed H1N1, SAR-CoV-2 infection and co-infection with both viruses.

\section{Methods}

\subsection{Study population}

This retrospective study analyzed patients admitted to the Guangzhou Eight People's Hospital with laboratory-confirmed H1N1 and COVID-19 infection. After excluding 7 patients who were minors ([?]18 years) and four patients with immune deficiency, a total of 220 H1N1-infected patients between January 2016 and May 2019 were enrolled in this study. For patients with COVID-19, we excluded 6 patients who were minors ([?]18 years) and three patients with immune deficiency, after which 285 COVID-19 patients admitted to the hospital between January 2020 and April 2020 were enrolled. Of these patients, 36 patients who had a mixed infection of H1N1 and COVID-19 were used as the comparator group. The study was approved by the Guangzhou Eight People's Hospital Medical Ethical Committee.

\subsection{Clinical data collection and definitions}

Electronic and written medical records were reviewed for all subjects. Data collected included demographic details, comorbid illnesses, presenting symptoms and signs, blood biochemical indexes, antiviral and antibiotic use, vasoactive drugs received (dobutamine or noradrenaline), intensive care unit (ICU) admission, hospital length of stay, requirement for ventilatory support, and occurrence of adverse events. Pneumonia was defined as the presence of pulmonary infiltrates on chest imaging not contributable to other causes. ARDS was defined based on the Berlin definition ${ }^{21}$. Immune deficiency was defined as organ transplant, active therapy for malignancy, and high-dose steroids/other iatrogenic drugs. Mixed infection of H1N1 and COVID-19 is defined as nucleic acid detection show positivity in both viruses, and the management measures refer to those of COVID-19 infection.

\subsection{Virus identification}

H1N1 virus infection were confirmed by analysis of nasopharyngeal swabs, sputum, bronchoalveolar lavage fluid and/or blood and/or urine samples using H1N1 virus Nucleic Acid Detection kit (Liferiver) ${ }^{22}$. SARS$\mathrm{CoV}-2$ infection were confirmed by analysis of nasopharyngeal swabs, sputum and blood samples using nucleic acid detection by RT-PCR ${ }^{23}$.

\subsection{Statistical analysis}

Continuous variables are described medians (interquartile ranges, IQRs). Categorical variables are presented as frequencies and percentages. Comparisons of proportions were performed with chi-square and Fisher's exact tests; continuous variables were compared using the Mann-Whitney U test. All probabilities were 2tailed, with statistical significance defined as $\mathrm{P}$ [?] .05. Binary logistic regression was performed to estimate the odds ratio (OR) and $95 \%$ confidence interval (CI) for comparison of clinical hospitalization outcomes in H1N1-infected, SAR-COV-2 infected and mixed infected groups. Adjustment factors included age, sex, comorbidities and smoking history. These risk factors were previously shown to be associated with the clinical prognosis of influenza and COVID-19 patients and served as confounders ${ }^{24,25}$. All analyses were performed using STATA, version 14.

\subsection{Role of the funding source}

The funders of the study had no role in the study design, data collection, data analysis, data interpretation, 
or writing of the report. The corresponding authors had full access to all the data in the study and had final responsibility for the decision to submit for publication.

\section{Result}

\subsection{Comparison between H1N1 infected patients and SAR-COV-2 infected patients.}

Demographic characteristics, ABO blood group and comorbidities prior to admission of all hospitalized patients are presented in Table 1 . The proportions of men and patient blood group were similar in the two groups. The median ages of H1N1 and SAR-COV-2-infected patients were 50.0 years (IQR 30.0-65.0, range 20.0-90.0) and 47.0 years (IQR 33.0-61.0, range 18.0-90.0), respectively ( $\mathrm{P}>.05$ ). The duration from disease onset to admission was longer in $\mathrm{H} 1 \mathrm{~N} 1$ patients (4.0 vs $3.0, \mathrm{p}=0.001$ ). As for comorbid conditions at admission, cerebrovascular disease, diabetes mellitus, hyperlipidemia and COPD were more common in H1N1-infected populations than those in SAR-COV-2-infected group. There were no significant differences between the groups in proportion of ABO blood group. Clinical symptoms were more frequently shown in H1N1-infected group than SAR-COV-2-infected group at admission except for dry cough and diarrhea. Diarrhea was more common in SAR-COV-2-infected group: $(4.02 \%$ vs $0.91 \%, \mathrm{P}=.033)$. The difference in the proportion of fever was the most obvious: $(82.73 \%$ vs $48.59 \%, \mathrm{p}<.001)$. In addition, H1N1 patients had higher admission temperature (median $37.8^{\circ} \mathrm{C}$, IQR $(36.8-38.2)$ vs. $\left.37.2(36.8-37.6) ; \mathrm{p}<.001\right)$. The comparison of the results of routine blood tests at admission are shown in table 1. For infection-related biomarkers, C-reactive protein level and procalcitonin level on admission were higher in H1N1 patients (median C-reactive protein level $44.96 \mathrm{mg} / \mathrm{L}$, IQR 20.36-77.75; median procalcitonin level $0.16 \mathrm{ng} / \mathrm{mL}[0.05-$ 0.61]) than non-ICU patients (median C-reactive protein level $32.08 \mathrm{mg} / \mathrm{L}$ [12.08-36.30], $\mathrm{p}<.001$; median procalcitonin level $0.07 \mathrm{ng} / \mathrm{mL}$ [0.04-24.7], $\mathrm{p}=0.0332)$.

As for treatment and clinical outcome on the two viruses, SAR-COV-2-infected patients were significantly more frequently received antiviral therapy ( $84.74 \%$ vs $76.82 \%, \mathrm{p}=.029)$ (table 2 ), as was oxygen therapy: $(62.65 \%$ vs $40.91, \mathrm{p}<0.001)$. Pneumonia and multilobar infiltrates were more frequent occurred in SARCOV-2-infected patients. SAR-COV-2-infected patients' median duration of hospital stay was longer than those of H1N1 infected patients: (17.5 days vs 7 days, $\mathrm{P}<.001)$. Generally, the rates of adverse clinical events were higher in elder patients (Figure 1). In univariable analysis, the odds of hospitalization outcomes (length of stay in hospital $>20$ days and pneumonia) in SAR-COV-2-infected patients were higher than in those hospitalized with $\mathrm{H} 1 \mathrm{~N} 1$ infection, albeit with a lower risk for highest temperature $>39^{\circ} \mathrm{C}$ during hospitalization. When adjusting for age, sex, comorbidities and smoking history our regression model showed similar results (Figure 2).

\subsection{Comparison betweenSAR-COV-2 infected patients andmixed-infected patients.}

When comparing SAR-COV-2 infected patients with mixed-infected patients (Table 1), we observed that mixed-infected patients $(\mathrm{n}=36)$ tended to be older (median 56.0 years vs 47.0 years, $\mathrm{p}=0.027$ ), with a higher proportion of patients with more than two comorbidities $(25.0 \%$ vs $8.84 \%, \mathrm{P}=.004)$. There were no major differences regarding sex or blood group. Myalgia was more frequent in mixed-infected patients; other symptoms showed no significant difference between the two groups. For comparison of the results of routine blood tests, neutrophil count and C-reactive protein level were significantly higher in mixed-infected patients (median neutrophil count $3.67 \times 109$ cells/L; median C-reactive protein level $51.2 \mathrm{mg} / \mathrm{L}$ ) than patients infected with SAR-COV-2 alone (median neutrophil count $2.83 \times 109$ cells/L, $\mathrm{P}=0.0081$; median C-reactive protein level $32.08 \mathrm{mg} / \mathrm{L}, \mathrm{P}<0.001)$ whilst haemoglobin and albumin were in a lower level.

Significantly more mixed-infected patients underwent vasoactive agents therapy and ventilation (table2). The length of hospital stay was longer (20.0 vs 17.5 days, $\mathrm{P}=0.02)$ and the rate of secondary bacterial infections ( $13.89 \%$ vs $3.21 \%, \mathrm{P}=0.004$ ), admittance to ICU (19.44\% vs $5.22 \%, \mathrm{P}=0.002)$ and mortality ( $2.78 \%$ vs $0, \mathrm{P}=0.008)$ were significantly higher in mixed-infected patients.

\section{Discussion}

With COVID-19-specific antiviral therapy advancing in clinical development, the question of differentiating 
SARS-CoV-2 infection from that of influenza and assessing their clinical impact in adult populations will likely become highly relevant to care decisions worldwide ${ }^{26}$. We reported here the clinical characteristics and severity of 505 patients hospitalized with illness caused by H1N1, SAR-COV-2 or mixed-infection of both viruses. H1N1-infected patients more frequently showed clinical symptoms than patients with SARSCoV-2 infected alone, especially for fever. During hospitalization, COVID-19 patients were more likely to develop pneumonia than H1N1 patients. Compared with infected by single virus, mixed-infection affected a predominantly older age group and was associated with poorer clinical outcome.

For the comparison between patients with H1N1 or SARS-CoV-2 infected alone, the symptoms commonly observed in both study groups included fever, dry cough, expectoration, and chill. We found that fever and dry cough were the dominant symptoms in two groups. Except for dry cough and diarrhea, symptoms were more frequent in H1N1 group. The absence of fever in COVID-19 was more common than in H1N1 influenza, moreover, higher body temperature was collected in H1N1 patients at admission, thus specified surveillance case definition on fever may facilitate identification of viral types of infection at admission. Compared with the H1N1 group, patients with SARS-CoV-2 infection had fewer prominent upper respiratory tract signs and symptoms (eg, expectoration or sore throat), indicating that the target cells might be located in the lower airway ${ }^{27}$. Correspondingly, SARS-CoV-2 group had a higher probability of pneumonia (OR:3.22, 95\%CI: 2.16-4.80) compared to H1N1 group, which further confirmed a difference in viral tropism between two viruses ${ }^{28,29}$. Consequently, SARS-CoV-2-caused pneumonia may account for the prolonged duration of hospitalization. The pathogenic mechanism underlying these differences between the two viruses are not clear yet and warrants further virological research. Of note, 10 (4.02\%) patients in SARS-CoV-2 group had diarrhea as an initial symptom which was more common than the H1N1 group (0.91\%); these findings echo a previous report ${ }^{13}$, and collectively suggesting this possibly a distinguishing clinical symptom of COVID-19. In the current study, laboratory test findings of H1N1 group at admission tended to be more abnormal, especially concerning measurements of inflammatory markers (eg. C-reactive protein level and Procalcitonin level). These findings were in line with the study conducted by Li Y et al. ${ }^{12}$; and can be attributable to the more common acute upper respiratory tract infection in hospitalized H1N1 patients ${ }^{30}$. This could explain the reason we have observed more symptoms of acute infection in H1N1 patients at admission. The diverse characteristics of infection provides the rationale for advancing antiviral interventions in efforts to improve outcomes of these two types of patients.

Of the 285 COVID-19 patients analyzed, 36 (12.6\%) patients were detected mix-infected by A(H1N1)pdm09. Co-infections more frequently occurred in patients with older age and more comorbidities, which may due to the relative lack of immunity to respirovirus in this population ${ }^{31}$. CRP and PCT, which was the severity index of pneumonia ${ }^{32}$, were lower in those with COVID-19 than the co-infection patients, suggesting co-infection is more severe than SARS-CoV-2 infected alone. Furthermore, after adjusting age, sex, comorbidities and smoking history, co-infection was associated with higher odds of several adverse clinical outcomes. This finding was in accordance with a previous report on other respiratory disease ${ }^{18}$. Thence, it's recommended that COVID-19 patients be detected for H1N1 infection at admission, and treat the detected mix-infected patients with rigorous clinical surveillance.

Secondary bacterial infection is a common and serious complication of influenza ${ }^{33,34}$. In this study, a higher rate of secondary bacterial infections was recorded among mix-infected patients. Therefore, similar to the recommendation for community-acquired pneumonia, it's reasonable for adults with COVID-19 who test positive for influenza to take initial antibacterial treatment, because it is difficult to exclude the presence of bacterial co-infection in these patients ${ }^{35}$.

There are limitations in this study. It was a single-center analysis, the features of the setting may not be representative of Chinese patients as a whole. As a retrospective study, the further clinical progression could not be collected. We also failed to explore the relationships regarding 30-days mortality due to the limited number of death cases $(\mathrm{n}=2)$.

In conclusion, H1N1 patients more frequently showed clinical symptoms than COVID-19 patients, especially for fever, which may provide recommendations for initial differential diagnosis. In contrast, COVID-19 
patients have a higher incidence of pneumonia than H1N1 patients during hospitalization. Mixed-infection affected a predominantly older age group and was associated with poorer clinical outcomes. So far, we do not yet have a COVID-19 vaccine, but safe and moderately effective influenza vaccines are available. Thus, a widespread use of influenza vaccines is important now than ever to reduce the risk of co-infection. Additionally, molecular diagnostic testing for both viruses is recommended for all patients with acute respiratory illness which would enable patients with co-infection to be recognized and given appropriate treatments in time in efforts to improve outcomes.

\section{Conflict of interests}

Authors have no conflict of interest to declare.

\section{Acknowledgments}

This research is supported by the Open Project of Guangdong Provincial Key Laboratory of Tropical Disease Research. We are indebted to all health-care workers involved in the diagnosis and treatment of patients in Guangzhou Eight People's Hospital.

\section{Reference}

1. Thommes EW, Kruse M, Kohli M, Sharma R, Noorduyn SG. Review of seasonal influenza in Canada: Burden of disease and the cost-effectiveness of quadrivalent inactivated influenza vaccines. Human vaccines \& immunotherapeutics. 2017;13(4):867-876.

2. Lozano R, Naghavi M, Foreman K, et al. Global and regional mortality from 235 causes of death for 20 age groups in 1990 and 2010: a systematic analysis for the Global Burden of Disease Study 2010. Lancet (London, England). 2012;380(9859):2095-2128.

3. Jilani TN, Jamil RT, Siddiqui AH. H1N1 Influenza (Swine Flu). In: StatPearls. Treasure Island (FL): StatPearls Publishing Copyright (C) 2020, StatPearls Publishing LLC.; 2020.

4. Nickol ME, Kindrachuk J. A year of terror and a century of reflection: perspectives on the great influenza pandemic of 1918-1919. BMC Infect Dis. 2019;19(1):117.

5. Shoubaki LA. Surveillance snapshot: Summary of the Department of Defense Global Respiratory Pathogen Surveillance Program, 2017-2018 influenza season. Msmr. 2018;25(10):22.

6. Saunders-Hastings PR, Krewski D. Reviewing the History of Pandemic Influenza: Understanding Patterns of Emergence and Transmission. Pathogens (Basel, Switzerland). 2016;5(4).

7. WHO announces COVID-19 outbreak a pandemic. 2020; http://www.euro.who.int/en/healthtopics/healthemergencies/. Accessed June 20.

8. "World Health Organization." Daily data on coronavirus (COVID-19). 2020. 2020; https://covid19.who.int/. Accessed June 24.

9. Yu H, Alonso WJ, Feng L, et al. Characterization of regional influenza seasonality patterns in China and implications for vaccination strategies: spatio-temporal modeling of surveillance data. PLoS medicine. 2013;10(11):e1001552.

10. Chauhan RP, Gordon ML. A Systematic Review Analyzing the Prevalence and Circulation of Influenza Viruses in Swine Population Worldwide. Pathogens (Basel, Switzerland). 2020;9(5).

11. Konala VM, Adapa S, Gayam V, et al. Co-infection with Influenza A and COVID-19. European journal of case reports in internal medicine. 2020;7(5):001656.

12. Li Y, Wang H, Wang F, et al. Comparison of Hospitalized Patients with pneumonia caused by COVID19 and influenza A in children under 5 years. International journal of infectious diseases : IJID : official publication of the International Society for Infectious Diseases. 2020. 
13. Yin Z, Kang Z, Yang D, Ding S, Luo H, Xiao E. A Comparison of Clinical and Chest CT Findings in Patients With Influenza A (H1N1) Virus Infection and Coronavirus Disease (COVID-19). AJR American journal of roentgenology. 2020:1-7.

14. Yue H, Zhang M, Xing L, et al. The epidemiology and clinical characteristics of co-infection of SARSCoV-2 and influenza viruses in patients during COVID-19 outbreak. Journal of medical virology. 2020.

15. Ozaras R, Cirpin R, Duran A, et al. Influenza and COVID-19 Co-infection: Report of 6 cases and review of the Literature. Journal of medical virology. 2020.

16. Cuadrado-Payán E, Montagud-Marrahi E, Torres-Elorza M, et al. SARS-CoV-2 and influenza virus co-infection. Lancet (London, England). 2020;395(10236):e84.

17. Lai CC, Wang CY, Hsueh PR. Co-infections among patients with COVID-19: The need for combination therapy with non-anti-SARS-CoV-2 agents? Journal of microbiology, immunology, and infection = Wei mian yu gan ran za zhi. 2020 .

18. Alfaraj SH, Al-Tawfiq JA, Alzahrani NA, Altwaijri TA, Memish ZA. The impact of co-infection of influenza A virus on the severity of Middle East Respiratory Syndrome Coronavirus. The Journal of infection. 2017;74(5):521-523.

19. Gregianini TS, Varella IRS, Fisch P, Martins LG, Veiga ABG. Dual and Triple Infections With Influenza A and B Viruses: A Case-Control Study in Southern Brazil. The Journal of infectious diseases. 2019;220(6):961968.

20. Redford PS, Mayer-Barber KD, McNab FW, et al. Influenza A virus impairs control of Mycobacterium tuberculosis coinfection through a type I interferon receptor-dependent pathway. The Journal of infectious diseases. 2014;209(2):270-274.

21. Ranieri VM, Rubenfeld GD, Thompson BT, et al. Acute respiratory distress syndrome: the Berlin Definition. Jama. 2012;307(23):2526-2533.

22. Zou X, Chang K, Wang Y, et al. Comparison of the Cepheid Xpert Xpress Flu/RSV assay and commercial real-time PCR for the detection of influenza A and influenza B in a prospective cohort from China. International journal of infectious diseases : IJID : official publication of the International Society for Infectious Diseases. 2019;80:92-97.

23. Wang $\mathrm{D}, \mathrm{Hu} \mathrm{B}, \mathrm{Hu} \mathrm{C}$, et al. Clinical Characteristics of 138 Hospitalized Patients With 2019 Novel Coronavirus-Infected Pneumonia in Wuhan, China. Jama. 2020;323(11):1061-1069.

24. Zhou F, Yu T, Du R, et al. Clinical course and risk factors for mortality of adult inpatients with COVID-19 in Wuhan, China: a retrospective cohort study. Lancet (London, England). 2020;395(10229):1054-1062.

25. Louie JK, Acosta M, Winter K, et al. Factors associated with death or hospitalization due to pandemic 2009 influenza A(H1N1) infection in California. Jama. 2009;302(17):1896-1902.

26. Xing Q, Li G, Xing Y, Chen T, Li W, Ni W. Precautions are needed for COVID-19 patients with coinfection of common respiratory pathogens.2020.

27. Huang C, Wang Y, Li X, et al. Clinical features of patients infected with 2019 novel coronavirus in Wuhan, China. Lancet (London, England). 2020;395(10223):497-506.

28. Guan WJ, Ni ZY, Hu Y, et al. Clinical Characteristics of Coronavirus Disease 2019 in China. The New England journal of medicine. 2020;382(18):1708-1720.

29. Cao B, Li XW, Mao Y, et al. Clinical features of the initial cases of 2009 pandemic influenza A (H1N1) virus infection in China. The New England journal of medicine. 2009;361(26):2507-2517.

30. Kaul D, Rathnasinghe R, Ferres M, et al. Microbiome disturbance and resilience dynamics of the upper respiratory tract during influenza A virus infection. Nat Commun. 2020;11(1):2537. 
31. Gorse GJ, Donovan MM, Patel GB, Balasubramanian S, Lusk RH. Coronavirus and Other Respiratory Illnesses Comparing Older with Young Adults. The American journal of medicine. 2015;128(11):1251.e12111220 .

32. Ruan Q, Yang K, Wang W, Jiang L, Song J. Clinical predictors of mortality due to COVID-19 based on an analysis of data of 150 patients from Wuhan, China. Intensive care medicine. 2020;46(5):846-848.

33. Bal A, Casalegno JS, Melenotte C, et al. Influenza-induced acute respiratory distress syndrome during the 2010-2016 seasons: bacterial co-infections and outcomes by virus type and subtype. Clinical microbiology and infection : the official publication of the European Society of Clinical Microbiology and Infectious Diseases. 2020 .

34. Dawood FS, Chaves SS, Pérez A, et al. Complications and associated bacterial coinfections among children hospitalized with seasonal or pandemic influenza, United States, 2003-2010. The Journal of infectious diseases. 2014;209(5):686-694.

35. Metlay JP, Waterer GW, Long AC, et al. Diagnosis and Treatment of Adults with Community-acquired Pneumonia. An Official Clinical Practice Guideline of the American Thoracic Society and Infectious Diseases Society of America. American journal of respiratory and critical care medicine. 2019;200(7):e45-e67.

\section{Figure legends}

Figure 1. Adverse events' rates by patient subgroup

Figure 2 Forrest plot of the impact of infection type on the illness severity and outcomes of hospitalized patients in China.SARS-CoV-2=severe acute respiratory syndrome coronavirus 2.

\section{Hosted file}

Table.docx available at https://authorea.com/users/337040/articles/462717-clinical-featuresseverity-and-outcome-of-adult-inpatients-with-single-and-dual-infections-with-influenzaa-h1n1-and-sars-cov-2-a-retrospective-study-in-china
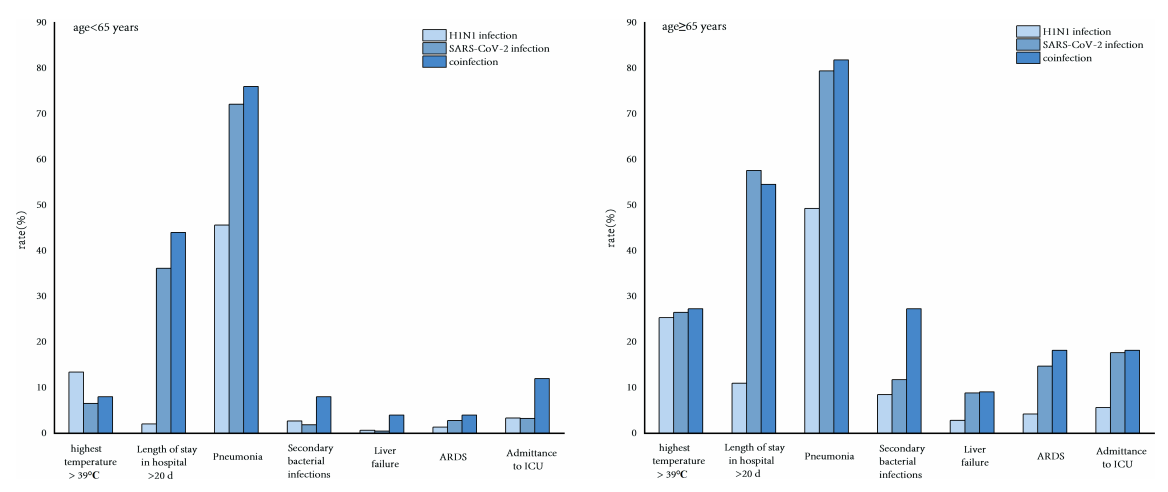


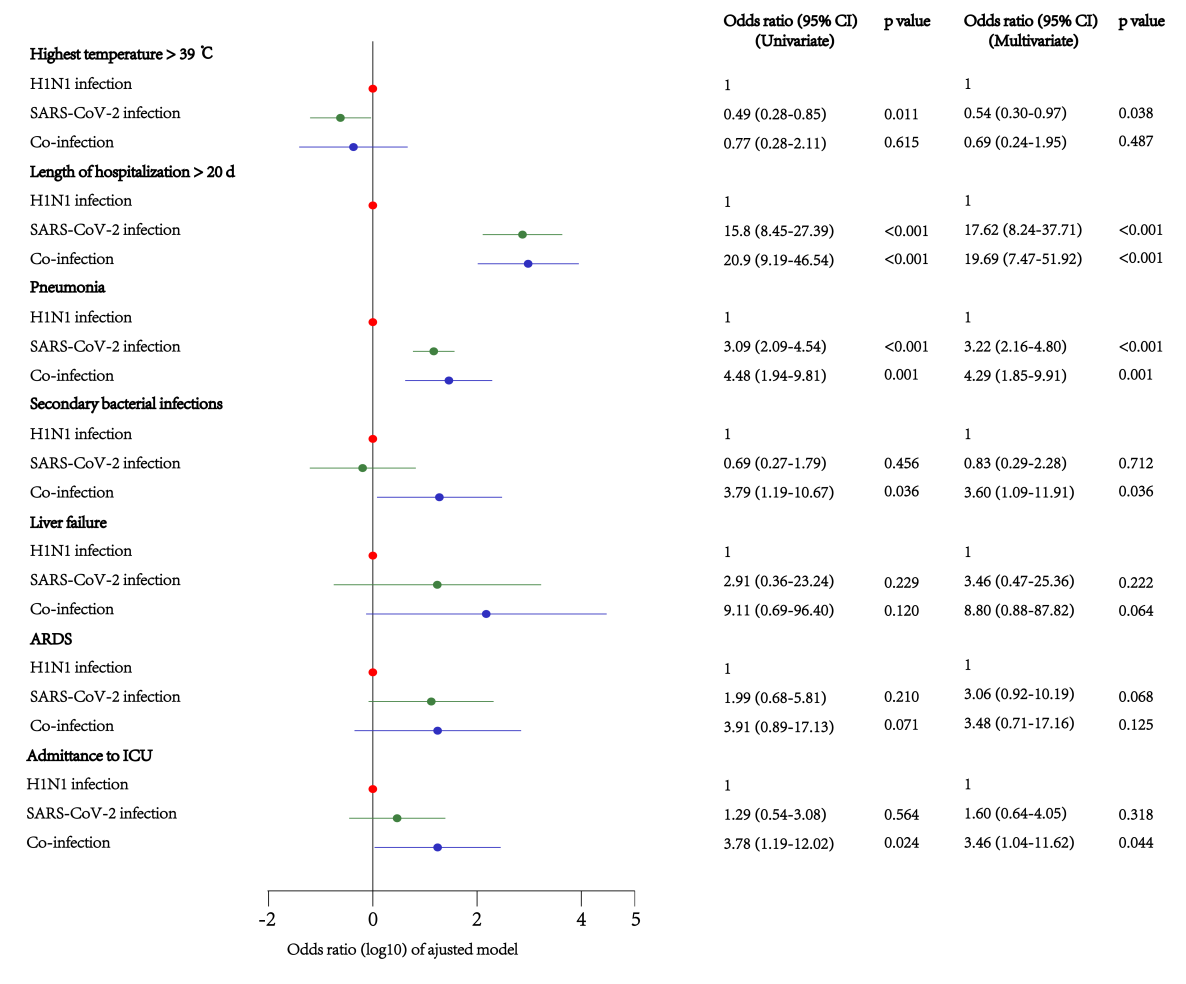

